\title{
Uma abordagem didática para a pressão interna de foguetes de garrafa PET propulsionados pela reação química entre vinagre e bicarbonato de sódio
}

A didactic approach to the internal pressure of PET bottle rockets powered by chemical reaction between vinegar and sodium bicarbonate

\author{
Marcus Vinicius Snovarski Fonseca ${ }^{*}\left[\right.$, Igor Matheus Leal Rodrigues ${ }^{1}$, Marcelo Belchior Snovarski \\ Fonseca $^{2}$
}

${ }^{1}$ Instituto Federal de Educação, Ciência e Tecnologia Farroupilha, São Vicente do Sul, RS, Brasil

${ }^{2}$ Universidade Federal do Pampa, Alegrete, RS, Brasil

Recebido em 08 de Novembro, 2017. Revisado em 01 de Março, 2018. Aceito em 05 de Março, 2018.

\begin{abstract}
O objetivo deste artigo é apresentar a busca de uma relação matemática entre a quantidade de reagentes e a pressão interna em foguetes de garrafa PET adequados ao nível IV da Mostra Brasileira de Foguetes. Inicialmente, foi descrito o dióxido de carbono produzido na reação entre o vinagre e o bicarbonato de sódio, através da equação de Clausius-Clapeyron. Os resultados previstos por esta abordagem não concordaram com os valores experimentalmente obtidos. Posteriormente, adotou-se a equação dos gases de van der Waals para descrever o comportamento do gás. Deste amplo estudo, observou-se que a última abordagem é capaz de descrever o comportamento da pressão no regime entre 0 - 60PSI. Acima de 60PSI nenhuma das abordagens descreveu a pressão de forma satisfatória. Consequentemente, entendeu-se que o aumento na pressão interna desloca o equilíbrio químico para o lado dos reagentes (Princípio de Le Chatelier) e concluiu-se que é possível obter uma equação empírica, que vincula a quantidade de reagentes e a pressão interna se for adotado um fator de atenuação dependente da pressão no número de mols de gás produzidos. Este estudo pode ser implementado como recurso de integração dos conteúdos abordados no ensino médio nas disciplinas de Física e Química.
\end{abstract}

Palavras-chave: Foguete de Garrafa PET, Equação de Clausius-Clapeyron, Equação dos gases de van der Waals.

The aim of this article is to present the search of a mathematical relation between the amount of reagents and the internal pressure on PET bottle rockets suitable to the level IV of the Brazilian Rocket Exhibition. Initially, it was described the dioxide carbon produced by the chemical reaction between the vinegar and the sodium bicarbonate through Clausius-Clapeyron equation. The predicted results from this approach didn't match from those obtained experimentally. Subsequently, it was adopted the van der Waals gas equation to describe the gas behavior. According to this wide study, it was observed that the last approach is able to describe the pressure behavior in the regime between $0-60 \mathrm{PSI}$. Above 60PSI none of the theoretical purposes were able to describe the internal pressure behavior. Consequently, it was understood that the increasing of internal pressure moves the chemical equilibrium to the side of reagents (Le Chatelier's Principle) and it was concluded that is possible to obtain an empirical relation between the pressure and amount of reagents, if it is assumed an attenuation pressure dependent factor in the mols number produced by the chemical reaction. This study can be implemented as an integration resource of the contents approached in high school mainly Physics and Chemistry.

Keywords: PET Bottle Rockets, Clausius-Clapeyron equation, van der Waals gas equation.

\section{Introdução}

Novas metodologias de ensino tornam-se cada vez mais necessárias à medida que os jovens alunos do ensino médio têm sido imersos em um cenário caracterizado por uma acessibilidade à informação sem precedentes na história da humanidade. Essa acessibilidade trouxe consigo uma inevitável mudança de visão sobre a figura do professor no ambiente escolar. Atualmente, ele não é mais visto

\footnotetext{
*Endereço de correspondência: marcus.fonseca@iffarroupilha.edu.br
}

como o único detentor do saber e o único participante ativo em sala de aula [1]. Os alunos do mundo conectado buscam informações e aprendem através de várias fontes, quase que exclusivamente em meios digitais, e o fazem apenas para aqueles assuntos pelos quais despertaram interesse. Porém, tornar o assunto abordado interessante ao ponto de vista do aluno atual é ir além da busca de um elo entre o que lhe é apresentado em sala de aula e a sua vida real, seu cotidiano. É necessário que o aluno sinta-se desafiado e intrigado com o que lhe é apresentado e essa etapa do processo de ensino-aprendizagem torna-se, mais 
do que nunca, um dos desafios mais fundamentais dentro da atividade docente [2].

Um dos recursos que podem ser explorados, com o objetivo de auxiliar o professor nesse desafio, são as feiras e mostras científicas, como a Mostra Brasileira de Foguetes (MoBFog). Essa mostra é um evento que ocorre, anualmente, como uma atividade paralela à Olimpíada Brasileira de Astronomia e Astronáutica (OBA), ambas organizadas pela Agência Espacial Brasileira (AEB) e pela Sociedade Astronômica Brasileira (SAB). Esses dois eventos têm como principal objetivo despertar o interesse e a vocação científica de alunos do ensino básico para a área das ciências, mais especificamente para a astronomia e para a engenharia de foguetes. A OBA consiste em provas com questões discursivas e de múltipla escolha, enquanto na MoBFog os alunos são instigados a projetar e desenvolver um foguete constituído de diferentes materiais e propulsionado de diferentes formas, conforme o nível de escolarização dos envolvidos. Os resultados obtidos individualmente pelos alunos, no caso da OBA, e aqueles obtidos pelas equipes, o alcance máximo horizontal dos foguetes, na MoBFog, são comparados com os demais alunos e equipes participantes de todo o Brasil para a distribuição das premiações.

No que se refere aos alunos do ensino médio, caracterizados como nível IV dentro da MoBFog, o projeto deve englobar a construção de uma base de lançamento e de um foguete. A base de lançamento pode ser confeccionada utilizando-se qualquer tipo de material, enquanto que o foguete deve ser obrigatoriamente construído com garrafas PET de quaisquer capacidades volumétricas. A propulsão do foguete deve ocorrer através da mistura de vinagre comercial com bicarbonato de sódio. No vinagre comercial está presente o ácido acético na concentração de $4 \%$ e a reação química desse ácido com o bicarbonato de sódio libera, como um de seus produtos, o dióxido de carbono, que pressuriza o sistema base-foguete. Uma vez pressurizado o sistema, a equipe de alunos deve ser capaz de realizar o lançamento acionando o dispositivo de trava do foguete (gatilho) a distância, buscando o maior alcance horizontal possível.

Vislumbra-se, dessa forma, um verdadeiro mix de conceitos para a elaboração e execução de um projeto de foguete nível IV da MoBFog. Sempre que esse entrelaçamento de conceitos é detectado, surge a possibilidade de que os conteúdos sejam abordados de uma forma interdisciplinar, reduzindo o caráter fragmentado do currículo escolar e possibilitando que a construção do conhecimento possa ser estabelecida a partir da articulação de diferentes saberes [3]. No caso específico da MoBFog, os alunos envolvidos devem aprofundar seus conhecimentos em assuntos que são geralmente trabalhados durante os dois primeiros anos do ensino médio, nas disciplinas de Física e Química. Na área da Física são necessários os conceitos abordados no estudo do movimento oblíquo, das leis de Newton, do momento linear e da conservação do momento linear, da pressão, da equação da continuidade e da teoria cinética dos gases [4]. Como a pressurização do sistema ocorre através de uma reação química é necessário que os alunos desenvolvam habilidades sobre a medida das quantidades de reagentes, concentração de reagentes, balanço e cálculo estequiométrico [5].

Consoante com essas ideias, Souza [6] mostrou que os conceitos físicos envolvidos no estabelecimento da uma relação matemática entre o ganho de velocidade do foguete, a massa ejetada e a pressão interna do sistema são aqueles abordados regularmente durante o ensino médio. São utilizados basicamente os conceitos de conservação da quantidade de movimento, centro de pressão e centro de massa, equação da continuidade e equação de Bernoulli [4]. Além disso, a análise desenvolvida pelo autor não requer recursos matemáticos mais avançados, como por exemplo, o uso de cálculo diferencial e integral [7]. Sendo assim, como sugere o próprio autor, os alunos de ensino médio possuem total capacidade de acompanhar e compreender os passos que o conduzem às suas conclusões. Nesse sentido, Calheiros e Palandi descrevem, em seu trabalho, o êxito obtido na experiência de desenvolver esse tipo de foguete com alunos do ensino médio de uma escola pública brasileira [8].

No entanto, o modelo de foguete analisado no estudo apresentado por Souza não se enquadra como os modelos aceitos no nível IV da MoBFog, visto que a pressurização do foguete é realizada mecanicamente através de uma bomba de ar. Além de não estar de acordo com o nível da MoBFog ao qual se enquadrariam os alunos do ensino médio, o fato de a pressão ser gerada mecanicamente excluiu a possibilidade de ser promovida uma integração dos conhecimentos da área da Física com os da Química. Quando a pressurização ocorre através do dióxido de carbono gerado na mistura entre o vinagre e o bicarbonato de sódio, o estudante deve também aplicar os conceitos trabalhados nas aulas de Química, mais especificamente os de cálculo estequiométrico, para conseguir obter a maximização do alcance horizontal do foguete construído.

Porém, mesmo sendo necessários apenas conceitos elementares de Física e Química, juntamente com uma matemática não muito avançada, não se encontra na literatura uma análise teórica ou empírica para o comportamento da pressão interna em relação à quantidade de reagentes presentes na cápsula de pressurização de foguetes do nível IV da MoBFog. Essa constatação foi verificada durante o desenvolvimento de recente projeto de pesquisa no Instituto Federal Farroupilha - Campus São Vicente do Sul, que tinha como objetivo determinar os pontos fundamentais que permitiriam a construção de uma base de lançamento e de um foguete com alcance horizontal superior a 200 metros. Nas análises realizadas no projeto, foi possível verificar que a pressão interna gerada pelo dióxido de carbono proveniente da reação química, é o principal fator ligado à velocidade de ejeção de massa da cápsula de pressurização e a velocidade de ejeção, por sua vez, determina a velocidade final do foguete no pro- 
cesso de aceleração. Assim, a pressão interna do sistema caracteriza-se como fator fundamental na maximização do alcance horizontal de um foguete desse tipo.

\section{Ensaios Experimentais e as Previsões Teóricas}

Como a pressão interna do sistema é o parâmetro fundamental na otimização do alcance horizontal, o presente estudo teve como primeiro passo a determinação experimental do comportamento do valor desta pressão em relação à quantidade de reagentes.

Nesse sentido, desenvolvemos um sistema simples para a medição da pressão interna. Esse sistema consiste em um manômetro conectado à tampa de uma garrafa PET, utilizando-se dois anéis de borracha para a vedação, e por um alfinete, colado no fundo da garrafa.

Inicialmente, adicionamos a quantidade escolhida de bicarbonato de sódio ao interior da garrafa. Posteriormente, colocamos um balão ou bexiga de festa no interior da garrafa e a quantidade de vinagre a ser utilizada dentro do balão. Fechamos o balão e vedamos a garrafa com a utilização da tampa na qual está instalada o manômetro. Uma leve agitação na garrafa faz o balão ser estourado pelo alfinete, permitindo o contato entre o vinagre e o bicarbonato de sódio. A pressão interna da garrafa pode ser lida diretamente pelo valor indicado no manômetro conforme indica o esquema de montagem da figura (1).

Essa estratégia para medição da pressão é baseada na forma como é medida a pressão interna do sistema basefoguete antes dos lançamentos. A única diferença está no fato de que, nos lançamentos, o manômetro está conectado à base de lançamento e o foguete está conectado a essa base através do bocal da garrafa PET.

A construção da curva experimental da pressão interna do sistema foi realizada alterando-se a quantidade

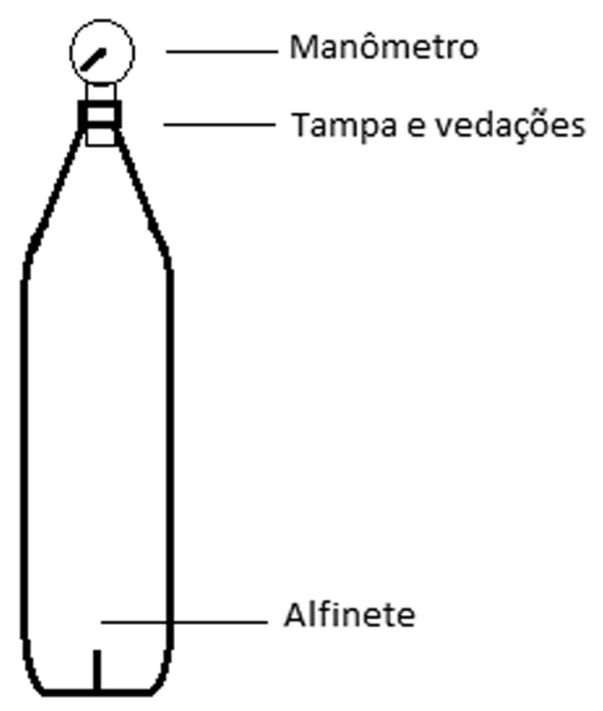

Figura 1: Dispositivo para determinação da pressão interna. de vinagre e de bicarbonato de sódio que eram utilizados em cada uma das medições. Os resultados dessas medições aparecem na figura (2). A unidade de medida da pressão mostrada na figura (2) foi escolhida baseada no fato de que o manômetro utilizado em nossas medições, bem como aqueles geralmente utilizados pelas equipes na MoBFog, são modelos comerciais que utilizam o PSI na sua escala de valores. Sendo assim, optamos por utilizar essa unidade em nossas análises e a conversão para a unidade do Sistema Internacional de Unidades pode ser feita através da relação:

$$
1 P S I=6,89 k P a
$$

\section{1. $\mathrm{O}_{2}$ como um gás ideal}

Uma vez obtido o comportamento experimental da pressão interna do foguete, surgiu a ideia de utilizar a aproximação de gás ideal para o dióxido de carbono produzido na reação e, através da equação de Clapeyron, relacionar os valores de volume, temperatura e quantidade de reagentes com o valor da pressão do sistema, ou seja, verificar se a equação de Clapeyron seria capaz de prever os valores de pressão encontrados nos ensaios. Para isso, inicialmente realizamos um estudo acerca da reação química entre o bicarbonato de sódio e o vinagre. Na mistura desses dois reagentes, o ácido acético $\left(\mathrm{CH}_{3} \mathrm{COOH}\right)$ presente no vinagre, em uma concentração de $4 \%$, reage com o bicarbonato de sódio $\left(\mathrm{NaHCO}_{3}\right)$. A reação química pode ser escrita na forma:

$$
\begin{aligned}
& \mathrm{CH}_{3} \mathrm{COOH}+\mathrm{NaHCO}_{3} \rightarrow \mathrm{CH}_{3} \mathrm{COONa} \\
& +\mathrm{CO}_{2}+\mathrm{H}_{2} \mathrm{O}
\end{aligned}
$$

É a reação acima que permitirá que sejam relacionadas a quantidade de reagentes presente no interior do foguete e a quantidade de dióxido de carbono produzido. Adotando que tanto o ácido acético como o vinagre possuem

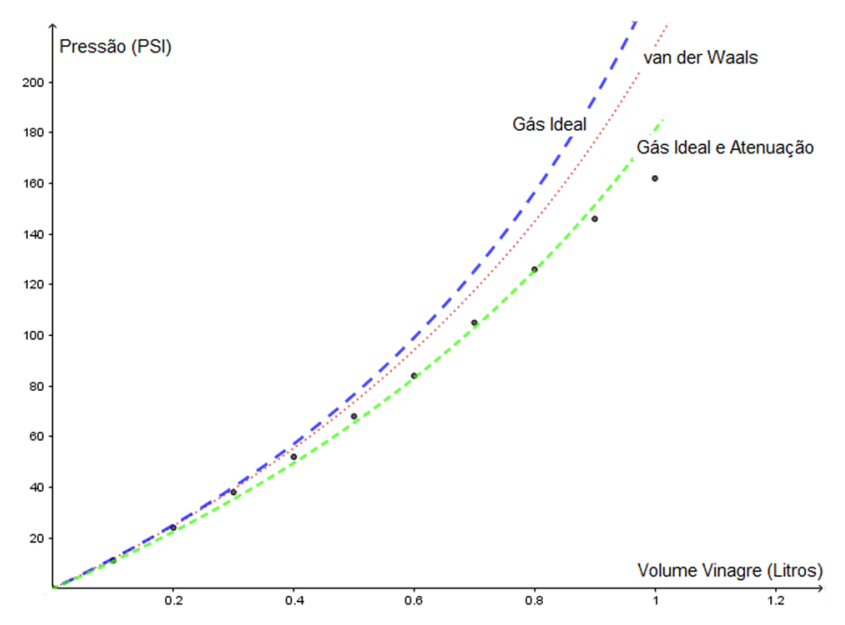

Figura 2: Confrontação entre os resultados teóricos e os obtidos em ensaios no Laboratório de Física do IFFarroupilha - Campus São Vicente do Sul. 
densidades com valor unitário e lembrando que esse ácido está presente em uma concentração de $4 \%$ no vinagre, pode-se concluir que cada litro de vinagre possuirá $40 \mathrm{~g}$ de ácido acético. Usando que a massa molar do ácido acético é $60 \mathrm{~g} / \mathrm{mol}$, pode-se relacionar a quantidade de mols de ácido com o volume de vinagre. Obtém-se, então:

$$
n_{\text {ácido }}=\frac{40}{60} V_{\text {vinagre }}=0,667 V_{\text {vinagre }}
$$

para o volume de vinagre, $V_{\text {vinagre }}$, expresso em litros.

A massa de bicarbonato de sódio, em gramas, necessária para que todo o ácido reaja, pode ser escrita na forma:

$$
m_{\text {bicarbonato }}=84 n_{\text {ácido }}=56,03 V_{\text {vinagre }}
$$

A reação (1) indica que para cada um mol de ácido reagido haverá a liberação de um mol de dióxido de carbono. Dessa forma, o número de mols de gás que serão liberados será calculado através da equação (2). Para a determinação do volume que será ocupado por esse gás, adotaremos que o valor pode ser obtido através da diferença entre o volume útil da garrafa e o volume de vinagre que foi adicionado. Isso significa supor que a adição do bicarbonato de sódio não altera, de forma significativa, o volume da mistura e, ainda, que o espaço livre dentro da garrafa não é ocupado por ar atmosférico. Com relação à temperatura, adota-se que, durante a reação química, não ocorrem trocas de energia através do calor que precisem ser consideradas, mantendo-se, assim, a temperatura dos reagentes igual à temperatura ambiente, $20^{\circ} \mathrm{C}(=293 \mathrm{~K})$. Todas essas suposições e seus possíveis efeitos serão discutidos em momento posterior. Por fim, considera-se o valor de $R=1,205 \frac{L . P S I}{\mathrm{~mol} . K}$ para a constante dos gases ideais, fazendo com que a equação de Clausius-Clapeyron tome a forma:

$$
P=235,5 \frac{V_{\text {vinagre }}}{V_{\text {garrafa }}-V_{\text {vinagre }}}
$$

para a pressão e os volumes expressos, respectivamente, em PSI e litros.

Os valores para a pressão, previstos pela equação acima, com relação à quantidade de reagentes, considerando-se uma garrafa PET com capacidade volumétrica útil de 2,105L (a conhecida garrafa de 2 litros), podem ser obtidos através de qualquer software apropriado (optou-se pela utilização do GeoGebra) e o resultado é apresentado na figura (2).

Pode-se claramente observar na figura (2) que, para baixos valores de pressão, existe uma boa concordância entre os valores previstos pela equação (3) e aqueles obtidos experimentalmente nos ensaios realizados no Laboratório de Física do Instituto Federal Farroupilha. Porém, à medida que a quantidade de reagentes vai aumentando, elevando a pressão interna do sistema, ocorre um afastamento entre o comportamento da pressão previsto e o experimental. Além do mais, existe uma discrepância muito grande entre os valores, justamente na faixa de interesse nos lançamentos, que fica entre 80 e 140PSI. Como será discutido mais adiante, nenhuma das suposições realizadas para a obtenção de equação (3) é capaz de fazer com que os seus resultados coincidam, de forma mais consistente, com os resultados experimentais.

Nesse momento é interessante salientar que a discrepância constatada poderia ser entendida com base no fato de que, ao aumentarmos a pressão interna do sistema através do aumento da quantidade de moléculas de gás presentes, afasta-se naturalmente o gás das suposições necessárias para a adoção do comportamento de gás ideal. Essa observação fez com que adotássemos um comportamento mais real para o dióxido de carbono do que aquele previsto pela aproximação de gás ideal. A partir desse momento, então, passa-se a tratar o dióxido de carbono através da equação dos gases de van der Waals.

\section{2. $\mathrm{O} \mathrm{CO}_{2}$ como gás de van der Waals}

No trabalho realizado por Costa [9] é apresentada uma discussão bastante completa sobre as diferenças entre as equações de Clausius-Clapeyron e de van der Waals. Com base nas conclusões obtidas pelo autor, surgiu a ideia de que a adoção da equação de van der Waals pudesse fazer os valores previstos para a pressão interna do sistema concordarem com os valores obtidos experimentalmente.

A equação de van der Waals tem a forma geral:

$$
\left(P+a \frac{n^{2}}{V^{2}}\right)(V-n b)=n R T
$$

As constantes $a$ e $b$ têm seus valores definidos com base no tipo de gás que se está tentando descrever. Para o caso do dióxido de carbono, essas constantes assumem valores $a=52,8 \frac{L^{2} \cdot P S I}{\mathrm{~mol}^{2}}$ e $b=0,043 \frac{\mathrm{L}}{\mathrm{mol}}[10]$.

Utilizando as mesmas suposições que adotamos na abordagem do gás ideal, bem como as relações encontradas entre o número de mols de dióxido de carbono e o volume de vinagre adicionado, e entre o volume útil da garrafa e o volume de vinagre, é possível novamente relacionar a pressão interna do sistema com a quantidade de reagentes, obtendo-se:

$$
\begin{aligned}
P & =235,5 \frac{V_{\text {vinagre }}}{V_{\text {garrafa }}-1,03 V_{\text {vinagre }}} \\
& -23,5 \frac{V_{\text {vinagre }}^{2}}{\left(V_{\text {garrafa }}-V_{\text {vinagre }}\right)^{2}}
\end{aligned}
$$

O comportamento previsto pela equação acima, considerando-se novamente uma garrafa com volume útil de 2,105L, é também apresentado na figura (2).

Observando-se a figura (2), é possível constatar que, até o momento, a descrição do dióxido de carbono através da equação de van der Waals é a que gera resultados mais próximos dos resultados experimentais. Porém, o aumento da pressão interna do sistema, decorrente do aumento da quantidade de reagentes, faz com que nem 
mesmo a equação (5) seja adequada para descrever o comportamento dos valores dessa pressão.

As primeiras discussões acerca da constatação acima devem residir, certamente, sobre o conjunto de suposições que adotamos para a descrição teórica do nosso sistema de estudo. Com relação a tais suposições, observa-se que nenhuma delas poderia fazer os resultados teóricos convergirem para os valores experimentais. Ao considerarmos que o volume livre da garrafa é preenchido por ar atmosférico à temperatura ambiente, teríamos um número adicional de moléculas de gás confinadas na cápsula de pressurização. Esse aumento no número de moléculas acarretaria o aumento no valor da pressão prevista, desviando ainda mais os valores teóricos dos experimentais. A mesma conclusão é obtida se considerássemos que a adição do bicarbonato de sódio aumenta o volume da mistura em relação ao volume de vinagre. Nesse caso, teríamos as moléculas de gás confinadas em um volume menor e, dessa forma, a pressão prevista seria ainda maior.

Por fim, ressaltamos que embora a reação química entre o ácido acético e o bicarbonato de sódio seja endotérmica, havendo absorção de calor e uma diminuição na temperatura dos produtos, a variação de temperatura é muito pequena para que os resultados teóricos sejam corrigidos ao ponto de concordarem com os resultados dos ensaios no Laboratório de Física. Assim podemos concluir que a discordância entre as previsões teóricas e os resultados experimentais não é consequência das suposições que adotamos no desenvolvimento acima apresentado.

\section{O Princípio de Le Chatelier e uma proposta empírica}

Como a equação de gás de van der Waals é uma equação de estado para gases reais, entendeu-se que a dificuldade de relacionar a quantidade de reagentes com a pressão interna, de forma a concordar com os resultados experimentais, não residia na relação entre o gás e a pressão que o mesmo exercia na cápsula. O problema da discordância está mais vinculado ao fato de que, embora tenhamos as proporções estequiométricas dos reagentes, esses não reagem completamente, devido justamente ao aumento da pressão interna. Essa afirmação se sustenta através do Princípio de Le Chatelier, o qual afirma que um aumento da pressão no ambiente onde a reação química ocorre faz com que o equilíbrio químico seja deslocado para o lado de menor volume [11]. No caso que estamos discutindo, o lado com menor volume é o lado dos reagentes e, assim, quanto maior for a pressão, mais deslocado para o lado dos reagentes estará o equilíbrio químico.

A constatação acima é pertinente para que entendamos o motivo da discordância entre os valores experimentais e os teóricos e para que possamos propor uma equação que seja capaz de descrever, de forma mais aproximada, a pressão interna do sistema com relação à quantidade de reagentes para o regime de interesse dos lançamen- tos. Nesse sentido, se o aumento da pressão desloca o equilíbrio químico da reação para o lado dos reagentes, diminuindo assim o número de mols de gás produzidos, adotou-se um atenuador nesse número de mols que, em nossa suposição, depende exclusivamente da pressão. Desse modo, quanto maior for a pressão do sistema, maior será a atenuação no número de mols de dióxido de carbono produzido. Além disso, adotamos uma relação tipo gás ideal para o dióxido de carbono, ou seja:

$$
P V=(n-\alpha P) R T,
$$

onde $\alpha$ é um parâmetro de ajuste a ser estabelecido após a comparação com os resultados experimentais.

$\mathrm{O}$ valor que melhor ajusta a curva ao regime de interesse é $\alpha=5,52 \times 10^{-4} \frac{\mathrm{mol}}{P S I}$, e, dessa forma, o comportamento da pressão em relação à quantidade de reagentes pode ser descrito através da expressão:

$$
P=235,5 \frac{V_{\text {vinagre }}}{\left(2,3-V_{\text {vinagre }}\right)},
$$

Os resultados previstos pela equação (7) são mostrados também na figura (2). Pode-se observar, na figura 2, que, para o regime de interesse dos lançamentos de foguetes de garrafa PET adequados ao nível IV da MoBFog, a equação (7) gera valores que estão de acordo com os valores experimentalmente obtidos, sendo, então, capaz de descrever o comportamento da pressão interna do sistema em relação à quantidade de reagentes.

É interessante salientar que poderia ter sido proposta, da mesma forma como ocorre na equação de van der Waals, uma correção de volume para a equação (6), através da adoção de um termo $(V-\beta)$. Porém, adotar essa correção terminaria por gerar uma equação que possuiria a mesma forma que a equação (7), mas com dois parâmetros livres $(\alpha$ e $\beta$ ). Se o interesse estiver sobre o comportamento da pressão para diferentes valores de temperatura, a adoção da correção de volume gera uma situação matemática mais completa e interessante.

\section{Considerações finais}

Destacamos, inicialmente, que o desenvolvimento apresentado acima não tem a intenção (e talvez o rigor metodológico necessário) de prever ou propor uma nova equação de estado térmica para um gás real. Buscou-se, muito mais, ancorá-la em uma base pedagógica e didática do que científica. Tentou-se descrever, de forma clara e objetiva, o conjunto de passos que foram dados na direção de obter-se poder de predição em relação à pressão interna da cápsula de pressurização de foguetes de garrafas PET, no sentido de auxiliar as equipes da MoBFog a desenvolverem seus projetos com mais controle sobre os parâmetros determinantes na maximização do alcance horizontal dos seus foguetes. Acreditamos que um desenvolvimento análogo possa ser desenvolvido com alunos do ensino médio (como foi o caso desse trabalho) tornando 
o aprendizado muito mais significativo e quebrando, de forma amena, o caráter fragmentado das ementas atuais das disciplinas da área das ciências exatas.

Em relação ao desenvolvimento apresentado, vimos que a equação de Clausius-Clapeyron relaciona, de forma satisfatória, a pressão interna do sistema com a quantidade de reagentes para valores de pressão até 60PSI. Nessa mesma região, a equação de van der Waals gera valores que se aproximam um pouco mais dos valores experimentais. Porém, nenhuma das duas abordagens permite que a pressão interna seja descrita consistentemente para valores superiores a 60PSI. Como a equação de van der Waals é uma equação para gases reais, entendeu-se que a discrepância encontrada não residia na descrição do gás e sim na diminuição da quantidade de mols de dióxido de carbono que são gerados na reação química, devido ao deslocamento do equilíbrio químico para o lado dos reagentes. Esse deslocamento, segundo o princípio de Le Chatelier, é decorrente do aumento da pressão no ambiente da reação. Sendo assim, foi proposto um fator de atenuação no número de mols de dióxido de carbono produzidos, que é exclusivamente dependente da pressão interna do sistema. Através dessa metodologia, foi possível encontrar uma equação que vincula, de forma satisfatória, a pressão interna dos foguetes de garrafa PET à quantidade de reagentes presentes no interior dos foguetes para o regime de valores de pressão de interesse dos lançamentos (80 - 140PSI).

\section{Agradecimentos}

Os autores agradecem aos revisores pelas importantes e significativas contribuições e ao $\mathrm{CNPq}$ pelo apoio financeiro fornecido ao longo do desenvolvimento do projeto.

\section{Referências}

[1] L.S. Alda, in: XII Seminário Internacional em Letras, Centro Universitário Franciscano, Pelotas, 2012, paper 4668.

[2] A.C.P. Fernandes, L.T.S. Auler, J.A.O. Huguenin e W.F. Balthazar, Revista Brasileira de Ensino de Física 38, e3504 (2016).

[3] M.A.A. Monteiro, I.C.C. Monteiro, E.L.S. Vaz e H.A. Acciari, in: V Simpósio Nacional de Ensino de Ciência e Tecnologia, Univ. Tecnológica Federal do Paraná, 2016, paper 01470092734.

[4] A. Gaspar, Compreendendo a Física (Ática, São Paulo, 2012) v. $2,1^{\circ}$ ed.

[5] A. Sardella, Química. Série novo Ensino Médio (Ática, São Paulo, 2012), $2^{\circ}$ ed.

[6] J.A. Souza, Física na Escola 8(2), 4 (2007).

[7] L. Leithold, O Cálculo com Geometria Analítica (Harba, São Paulo, 1994), v. $1,3^{\circ}$ ed.

[8] L.B. Calheiro e J. Palandi, Enseñanza de las Ciencias Núm. Extra, 574 (2013).

[9] D.M.A. Costa, Análise do Desvio de Comportamento Entre Gás Real e Gás Ideal, disponível em http://www2.ifrn.edu.br/ojs/index.php/HOLOS/ article/view/92

[10] N.H.T. Lemes, J.M. Oliveira e J.P. Braga, Quim. Nova 33, 1325 (2010).

[11] B.M. Mahan e R.J. Myers, Química: Um Curso Universitário (Edgard Blüncherm, São Paulo, 1995). 